\title{
NETGRAPH - AN EFFICIENT TOOL FOR PERIODIC TIMETABLING AND CAPACITY PLANNING
}

\author{
ZdenĚK Michl*, Michal DráBeK, Rudolf VÁvra \\ Department of Logistics and Management of Transport, Faculty of Transportation Sciences, CTU in Prague, \\ Czech Republic \\ * corresponding author: michlzde@fd.cvut.cz
}

ABstract. A netgraph is a graphical representation of a periodic timetable concept for given spatial or modal scope. It adds a third dimension to the network plan - time. Despite no strictly prescribed rules, a common practice notation has been settled over time. The authors propose an extension of a netgraph for planning of periodic railway capacity and operation, including related notation rules.

KEYWORDS: edge, netgraph, node, notation, periodic timetable, periodic capacity.

\section{INTRODUCTION}

A netgraph, also called interval graphic, is a graphical representation of a periodic (interval) timetable concept of public transport lines in a designated geographical or political territory, for a transport segment or a production unit. It displays at first traffic and transport coherence of the individual lines in transfer nodes and their service function on the territory (number of stops). Netgraph was made up of a combination of a network plan, as usually used in urban and local public transport, and a timetable, as used in railway timetabling. While the former reproduces the course of the lines and partially the intervals in the entire network, the latter shows in detail the scheduled traffic on a part of the network (mostly a single railway line). The netgraph adds a third dimension to the two-dimensional network plan - time schedule. Thus, in a netgraph, periodic timetable with small to medium intervals in an area can be clearly illustrated.

There are no strictly prescribed rules for creation of netgraphs, but over time a common practice notation has been settled. Since the level of detail, the purpose of creation and highlighted properties in netgraphs varies, it is always necessary to provide a legend of line (edge) types and colours and time notation used in it.

A netgraph may be created as an output of a specialised transport planning software package as german FBS - Fahrplanbearbeitungssystem (FBS/iPlan) [1], swiss Viriato [2] and others, enabling an overview of the constructed lines and connections at a glance for comparison. A basic netgraph can even be constructed using a spreadsheet office suite or another graphic tool.

A netgraph consists of following elements:

- nodes, including their names,

- edges, including the timetable notation for each public transport line and number of stops,

- technological processes,
- a legend.

Further, the authors propose an extension of a netgraph for planning of periodic railway capacity and operation. For such purpose, it is necessary to establish rules for notation of passing through defined station at a certain time, for alternative routing of a train path, for a train path for one direction only, and for crossing of the opposite trains on a single-track line. A proposal of related rules is presented and discussed consequently.

\section{Common Practice in Netgraph Notation}

There emerged two main producers of netgraphs iRFP. e. K. (Institut für Regional-und Fernverkehrsplanung), and SMA und Partner AG. Both companies provide timetabling software tools. Their rules for netgraph notation are mostly equal, except some differences (e.g. as in Figure 9 by SMA and as in Figure 10 by iRFP). Both companies publish on their websites a short introduction into purpose and notation of a netgraph. iRFP uses a term "interval graphic", SMA uses "netgraph". The authors of this paper have decided for the term "netgraph" for the sake of brevity. iRFP notes that a netgraph displays at least

- geographical offer: (public transport) "line network and line management, main railway stations, operated access points per line

- temporal offer: interval frequence per line as well as offer density on certain lines through overlap, arrival and departure times of the clocked lines in main railway and intermediate stations, determining of lines and transition periods in the stations

- qualitative offer: long-distance traffic and local transport services with regard to the lines, single products or train categories if applicable Train Operation Company, number of train changing per relation" 3 
SMA stresses that "a single line represents each service (and the corresponding journey in the reverse direction) connecting the stations it passes through on its trip, with the arrival and departure times for each station indicated.

The repeating aspect of the timetable means that a whole repeating family of trains can be covered by one line on the diagram. The connection times between services at a station can be seen at a glance, giving a powerful method for improving the coordination between services." [4]

The notation rules represented below generally come out from both above mentioned netgraph providers.

Besides FBS/iPlan and Viriato, some other timetabling software tools, e.g. RailSys [5], OptiTakt [6] and TAKT [7] can produce netgraphs as well.

\subsection{NODES}

Nodes of a netgraph represent important places of a transport network from the passenger's (user's) point of view. Each node must be named (in full or using an abbreviation - for the recommended place for descriptor see Figure 1). Optionally, a minimum transfer (connection) time in a node in minutes may be added to the description.

Node importance category, frequency of service and other attributes may be displayed using different borderline and filling types and colours.

A netgraph node depicts a timetable and network node, which is:

- station of origin or destination of at least one public transport line (or a part of its services),

- transfer (interchange) station to other public transport lines and transport systems, even if they are not included in the netgraph

- stations, where splitting or joining of trains or lines or other technology operation regularly takes place,

- other important places in the network, such as regional or tariff borders, crossing of trains etc., according to the purpose of creation of the netgraph.

\subsection{EDGES}

A netgraph edge represents a public transport line (or its branches and joint sections) from the passenger's point of view. Edges of lines passing more nodes are connected through the node boxes. Important edge attributes are line style and colour, which depicts the service function and interval of the public transport line.

\subsection{EdGe Colour}

Colours are usually used (according to the purpose of creation of the netgraph) for:

- transport segment of the line (e.g. train category),

- operation periods (e.g. lines operating only on working days, during peak hours etc.).

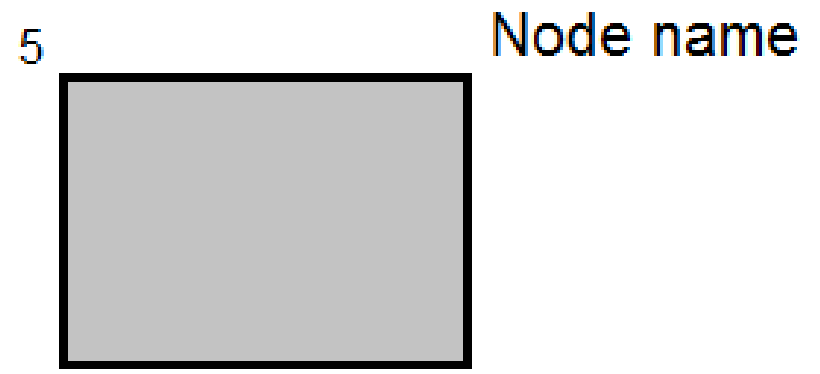

Figure 1. A node of a netgraph including name descriptor and the minimum transfer time (5 min).

- different transport modes (each mode in another colour),

- different train operating companies, public transport authorities etc., where applicable,

- in exceptional cases individual lines can be distinguished by the line colour.

There is no single correct way to colorize the lines. The selected scheme therefore has always to be explained in the legend next to the netgraph. Yet there are common conventions. The authors recommend getting inspired from corresponding use cases.

\subsection{Edge Line Style}

A line style of the edge distinguishes the interval between services of the line. Line style can even show public transport lines operated without a fixed interval. Generally, one solid line represents one service per period (usually one hour). By convention, the line styles as shown in Figure 2 are used. Different line styles are used e.g. in urban public transport systems. In each case, the line styles used in a netgraph should always be explained in the legend.

\subsection{Timetable Notation}

Edges representing public transport lines with periodic timetable are complemented with time labels. They indicate periodic arrival and departure times to and from each node, noted only in minutes. The descriptors are usually on the side of travel direction from the edge - on the right in most cases, on the left e.g. for Swiss railways. The descriptor closer to a node shows the arrival time, the one further from the node then the departure time respectively, as shown in Figure 3. Both lines (black and red) operate every hour between $\mathrm{A}$ and $\mathrm{C}$. Trains of the black line leave A every hour at minute 31 for $\mathrm{C}$. They arrive to B in minute 44 and leave in minute 45 , while arriving to $\mathrm{C}$ in minute 59. In the opposite direction the trains leave $\mathrm{C}$ every hour at minute 1 , call at $\mathrm{B}$ between minutes 15 and 16 and reach $\mathrm{A}$ in minute 29. Trains of the red line leaves A every hour at minute 4, does not call at $\mathrm{B}$ and arrive to $\mathrm{C}$ at minute 27 . In the opposite direction the trains of the red line leave $\mathrm{C}$ every hour in minute 33 , do not call at $\mathrm{B}$ and reach $\mathrm{A}$ in minute 56 . If trains of a public transport line 
a)

b)

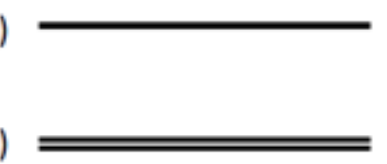

$2 \mathrm{~h}$ interval

$1 \mathrm{~h}$ interval

30 min interval d)

e)

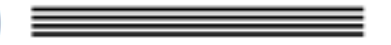

15 min interval

f)

non-perıodic service

FiguRE 2. Line style usage convention according to the service interval.

do not call at the node, the time descriptor is usually omitted also (see the red line at node B in Figure 3).

In some cases of netgraphs for timetabling or railway capacity planning purpose, it may also be useful to indicate the passage time as departure times. It is then useful to distinguish the nodes where the train calls with dots in the colour of the respective line at intersections of the edge and node borders (see Figure 4. This is also useful for lines with fixed stops but non-periodic timetable, where minute descriptors are omitted.

In the case of two-hours-interval the descriptor must also indicate the parity of the hour of operation (even or odd). By convention, the minute-descriptor of an even hour is descripted by normal font and that of an odd hour in italics, see Figure 5 The public transport line is operated every two hours between A and C. For $\mathrm{C}$ it leaves A every even hour at minute 40, arrives at B every odd hour in minute 14 , calls until minute 16 and arrives at $\mathrm{C}$ every odd hour in minute 59 . In the opposite direction, it leaves $\mathrm{C}$ every even hour at minute 01 , calls at $\mathrm{B}$ every even hour in minutes 44 to 46 and reaches A every odd hour in minute 20. Alternative resolution of even and odd hours is also possible, e.g. by underscoring the minute descriptor for an odd hour.

In the case of public transport lines operated every timetable period (60 minutes), there is no need to distinguish between odd and even hours, see both lines in Figure 4

For public transport lines operated more than once a timetable period (with notation as in Figure 2), only one minute-descriptor for all trains of the line within a period is provided, other service times of the period are omitted - see Figure 6] The public transport line is operated twice an hour (every 30 minutes) between nodes $\mathrm{A}$ and $\mathrm{C}$. For $\mathrm{C}$ it leaves A every hour in minute 03 and 33, arrives at B every hour in minute 29 and 59, calls there until minute 30 and 00 and arrives at $\mathrm{C}$ every hour in minute 58 and 28. In the opposite direction, it leaves $\mathrm{C}$ every hour in minute 02 and 32 , calls at B every hour in minutes 30 and 00 to 31 and 01 and reaches $\mathrm{A}$ every hour in minute 57 and 27. Descriptors for the opposite direction are calculated symmetrically along the main timetable axis (as complements to 60 or another timetable period).

\subsection{Number of Serviced Stops}

Intermediate stops of a public transport line are shown on the edge between nodes. Either each serviced stop is indicated with a solid dot (see Figure 7) or grouped in an empty circle with number of grouped stops next to it or in it (see Figures 8 and 9).

\subsection{Branching of a Public Transport LINE}

Branching (some trains continue from a node in other direction than the others) is indicated in the node with two connections between the edges, see Figure 10. Trains arrive from B to A every hour in minute 29. They continue every odd hour in minute 32 for $\mathrm{D}$, and every even hour in minute 32 for $\mathrm{C}$. The situation is similar in the opposite direction. The line style shows the doubled service interval of the branches.

\subsection{Splitting And Joining of Trains (UNITS)}

In this case, each train splits into two parts, and each part continues to a different destination, see Figure 11. Trains from B arrive in A every hour in minute 28 and split in two parts, one leaving for D every hour in minute 32 , the other one continuing to $\mathrm{C}$ every hour in minute 35 . The situation is similar in the opposite direction.

The form of linking of edges in a node can also indicate a U-Turn of trains of the public transport line.

\section{Proposed Additional Rules for Netgraph CREation AND Discussion THEREOF}

\subsection{Different Service Routing in Each DiRECTION}

In some cases, a route of a public transport line contains sections, where services operate in one direction only. Such sections are distinguished with arrows. Time descriptors are displayed in particular direction only. In Figure 12, the line is From A to $\mathrm{F}$ routed through nodes $\mathrm{B}, \mathrm{C}$ and $\mathrm{E}$, and in the opposite direction through nodes E, D and B.

\subsection{Alternative Routing of Freight TRAin Paths}

Lindner and von Redern [8] found that it was necessary to let periodic time windows ("canals") free for freight 


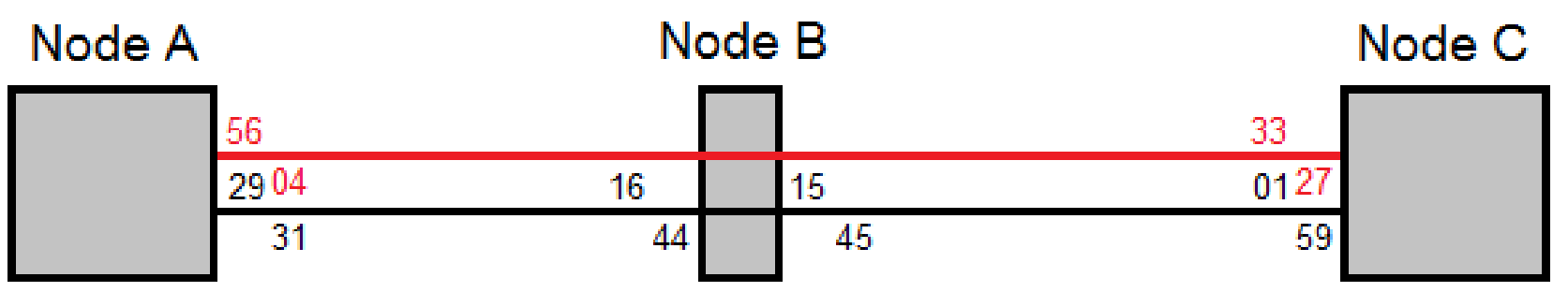

FiguRE 3. Example of timetable descriptors.

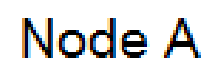

\begin{tabular}{|c|c|c|c|c|}
\hline \multicolumn{4}{|c|}{ Node B } & Node C \\
\hline 56 & 45 & & 33 & \\
\hline 2904 & 16 & 1516 & 0127 & \\
\hline 31 & 44 & 45 & 59 & \\
\hline
\end{tabular}

FiguRE 4. Extended timetable descriptors example. Serviced nodes are distinguished with dots of respective colour of the line.

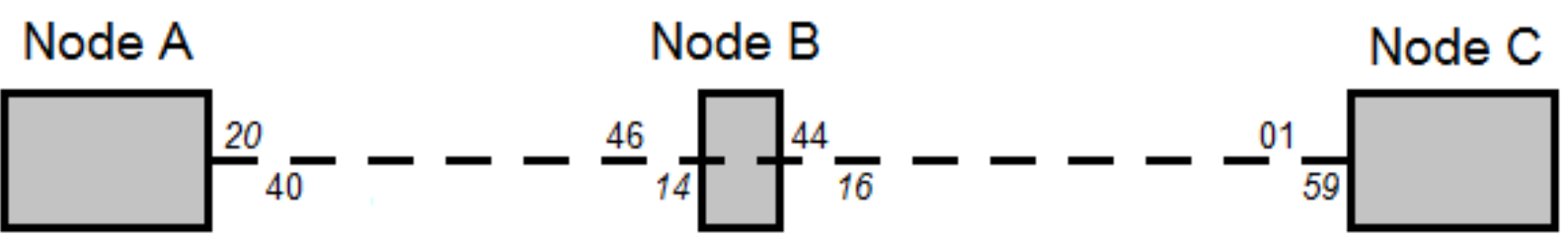

FiguRE 5. Extended timetable descriptors example $-2 \mathrm{~h}$ interval with odd hour distinguished.

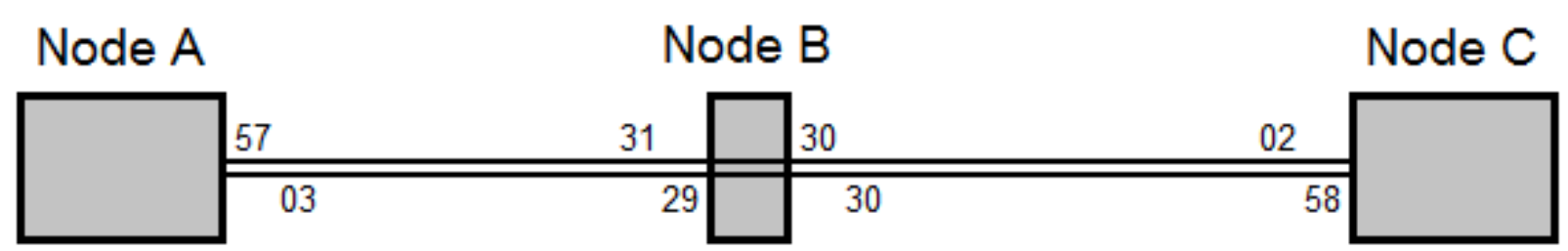

FiguRE 6. Extended timetable descriptors example - 30 min interval.

\section{Node A}

Node B

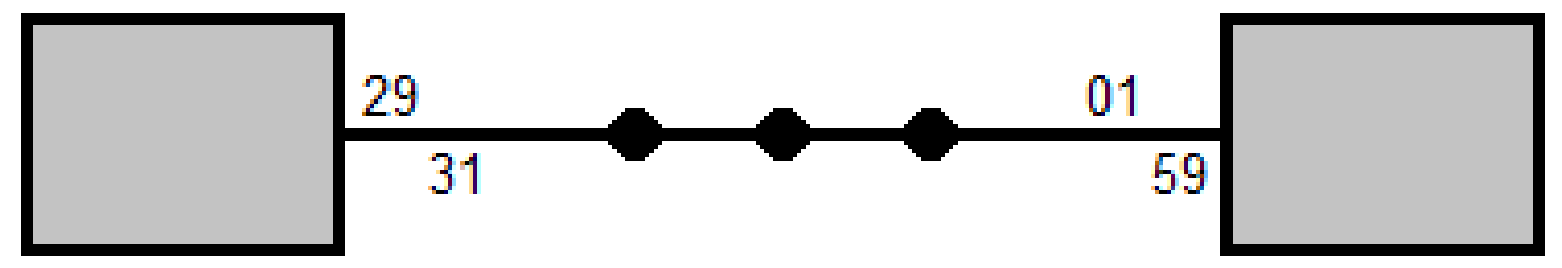

FiguRE 7. Three serviced intermediate stops between nodes A and B, each one indicated separately with a solid dot. 


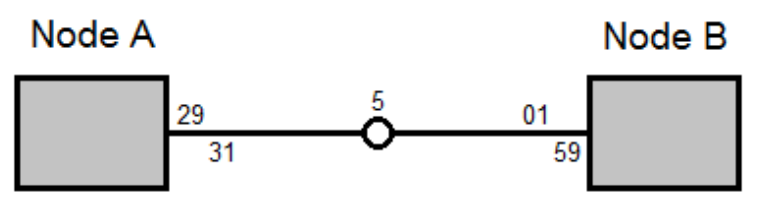

FiguRE 8. Five intermediate stops between nodes A and $\mathrm{B}$, the number of grouped stops is indicated with a numeric descriptor next to an empty circle.

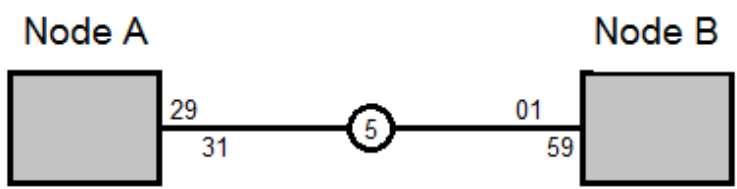

Figure 9. Five intermediate stops between nodes A and $\mathrm{B}$, the number of grouped stops is indicated with a numeric descriptor in an empty circle.

trains. These should be wide enough for required number of freight train paths per hour. These time windows should be preferably connected in nodes (if needed, among more than two railway lines to enable use by freight trains with various origin/destination stations). In the case of insufficient capacity, the authors proposed reviewing the structure of passenger transport offer.

This idea was further worked up by Drábek [9], who proposed a concept of network-bound periodic freight train paths with possible alternative routing in node station. This concept is, to a certain extent, an analogy to Integrated Periodic Timetable (IPT) in passenger transport. IPT is a network-wide, periodic offer of services and transfer connections between them. The concept of network-bound periodic freight train paths is a network-wide periodic offer of capacity that meets various requirements of freight train operating companies. Unified period and unified (zero) symmetry are maintained in this concept as well. Alternative routing through nodes represents an analogy to transfer connection in IPT. This alternative routing is illustrated in Figure 13. The train paths from B through $\mathrm{A}$ are routed alternatively in the direction of $\mathrm{C}$ and D. A freight train can pass through node A (without stop) in minute 32 , either in direction $\mathrm{C}$ or D. In the opposite direction, a freight train can pass through node $\mathrm{A}$ in minute 31, either from direction $\mathrm{C}$ or D.

\subsection{Train Crossing on a Single-track LINE}

For planning of periodic capacity of single-track lines, a key information is in which stations the trains in opposite directions periodically cross (meet) each other - at present day, or in a certain planning time horizon. In the case of IPT, a unified axis (minute) of symmetry is kept, and the crossing happens (at least approximately) in symmetry time. In Europe, a convention on zero symmetry is kept. Due to mathematical pre-

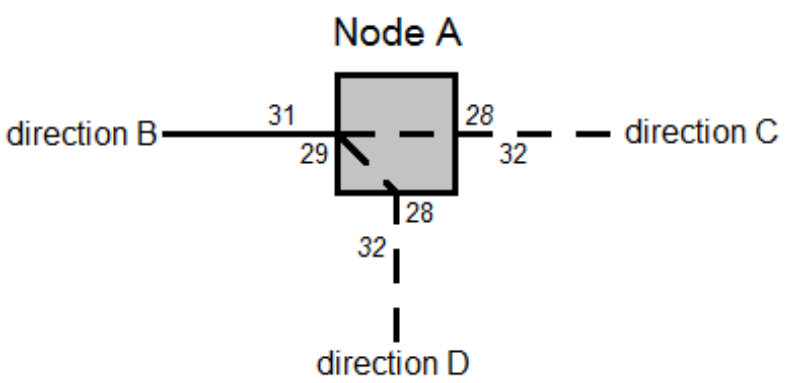

FiguRE 10. Branching of a public transport line into two directions.

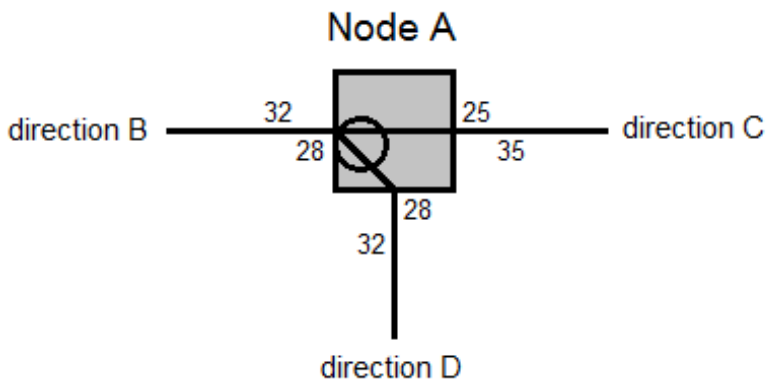

FIGURE 11. Splitting and joining of trains (units).

conditions of IPT, two groups of nodes with symmetry time always occur - for instance, for 60 min interval, zero symmetry means one group of nodes (stations) with symmetry time in minute 00 , and another group of nodes with symmetry time in minute 30 .

Necessity of displaying time descriptors in such symmetrical crossing stations depends on level of detail for the capacity planning. For timetabling purposes, the descriptors are necessary for information about (real or required) scheduled running time between neighbouring symmetry nodes. For conceptual planning, name of the node station may be sufficient, as such symmetrical crossing stations change only after implementation of a new IPT-stage (shortening of travel times).

Figure 14 shows, beyond a "common" netgraph, depiction of periodic crossing of fast train in station Katovice. As this crossing is not connected with a commercial stop and the netgraph serves for conceptual level of detail only, time descriptors for Katovice are omitted.

\subsection{Discussion}

Besides timetable concept elaboration and presentation, netgraphs can be used for railway capacity planning as well. The authors further propose additional rules to address various aspects of railway capacity planning. In many cases, the meaning of an edge, displayed in a netgraph, shifts from periodic service to periodic capacity, which may (fully or partially), or may not, be really used by a train (service). 


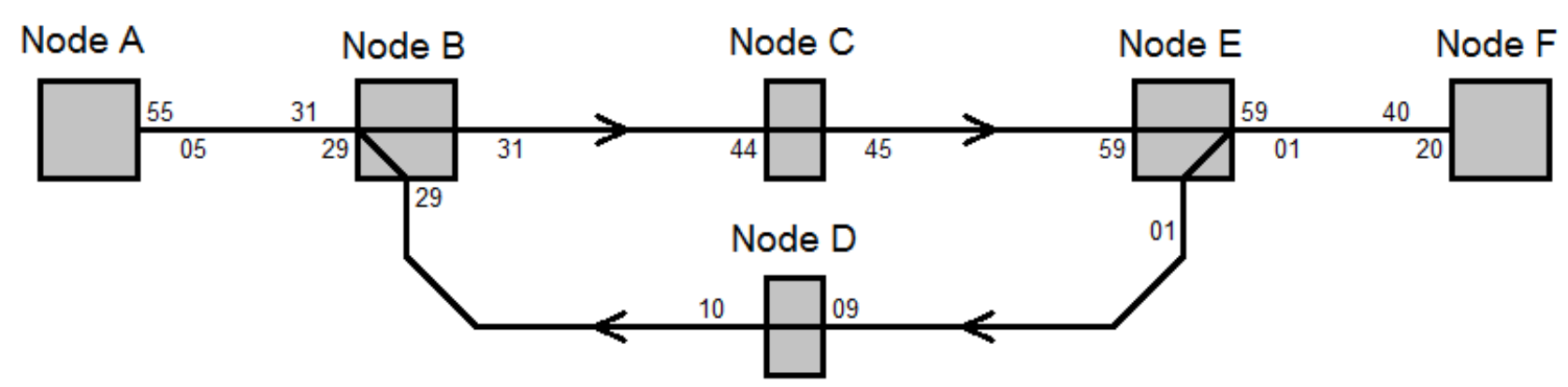

FiguRE 12. Operation of a public transport line in a certain direction only.

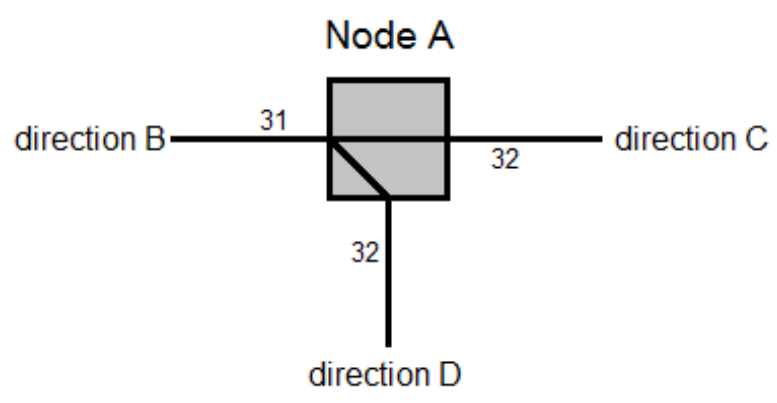

FiguRE 13. Alternative routing of freight trains (alternative train paths - from $\mathrm{A}$ either to $\mathrm{C}$ or $\mathrm{D}$ ).

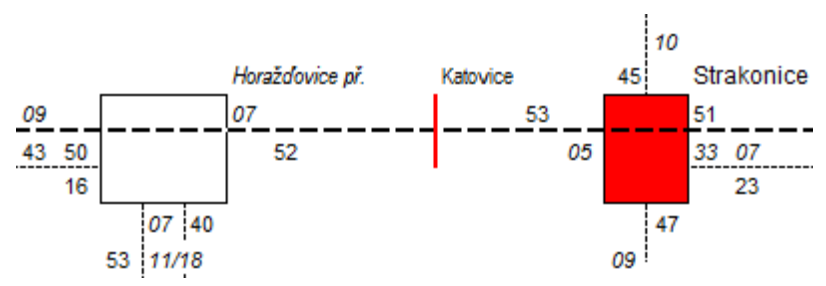

Figure 14. Example of descriptor of a crossing station (zero symmetry) [10.

\section{Conclusions}

A netgraph is a very useful tool not only for public transport (mostly railway) timetable conceptual planning. For periodic railway capacity planning (either periodic passenger service with half interval during the peak hours, or periodic capacity for freight trains, even with alternative routing). Netgraph cannot replace classical timetabling tools, but can enable an easy and rapid overview for network capacity planning. For codification of necessary descriptors of symmetrical periodic crossing in terms of group of symmetry nodes, all-day or peak only incidence and planning horizon, further research is needed.

\section{REFERENCES}

[1] iRFP e.K. Institut für Regional-und Fernverkehrsplanung. Our product: the timetable construction system fbs [online]. [cit. 2017-03-24] "http://irfp.de/our-product-fbs.html".

[2] S. und Partner AG: Viriato, ZLR. The versatile timetable system [online]. [cit. 2017-03-23] "http://www.sma-partner.com/en/solutions/viriatoand-zlr/overview".

[3] iRFP e.K. Institut für Regional-und Fernverkehrsplanung. Timetable creation [online]. [cit. 2017-03-23] "http://irfp.de/timetable-creation.html".

[4] S. und Partner AG: Viriato, ZLR. Netgraph. focussing on service [online]. [cit. 2017-03-23]

"http://www.sma-partner.com/en/solutions/viriatoand-zlr/functionality/netgraph".

[5] R. M. C. GmbH. Railsys [online]. [cit. 2017-03-17] "http://www.rmcon.de/railsys-en/".

[6] W. H. et al. Optitakt [online]. [cit. 2017-03-24] "http://www.optitakt.de/".

[7] C. o. T. F. s. U Dresden. Timetabling [online]. [cit. 2017-03-23] "https://tudresden.de/bu/verkehr/ila/vkstrl/forschung/ forschungsfelder/fahrplanung?set_language=en".

[8] H.-R. Lindner, H. von Redern. Güterz $\ddot{U} g e$ im Taktfahrplan - Möglichkeiten und Grenzen. Die Bundesbahn 10/1989.

[9] M. Drábek. Periodic freight train paths in network. doctoral thesis, 2014.

"http://takt.fd.cvut.cz/cargo/Drabek_thesis.pdf".

[10] M. Drábek. Netgraphs of regional railway public service in chosen Czech regions. CTU in Prague, undated. Unpublished. 